\title{
State relevance for model order reduction applied to a microgrid
}

\author{
Andrés Tomás-Martín, Aurelio García-Cerrada, Senior Member, IEEE, Lukas Sigrist, Member, IEEE, Sauro \\ Yagüe, Member, IEEE and Jorge Suárez-Porras
}

This work has been submitted to the IEEE for possible publication. Copyright may be transferred without notice, after which this version may no longer be accessible

\begin{abstract}
This paper presents a systematic model order reduction (MOR) algorithm based on state relevance applied to an islanded microgrid with electronic power generation. MOR of such islanded microgrids may not benefit, a priori, from the wellestablished time-scale separation usually applied to conventional power systems, and a systematic MOR is still an open issue. The proposed algorithm uses a balanced realization of the linear system, where state variables may not have physical meaning, to obtain the states' energies. It then calculates the relevance of the original system states from those energy values. The newly proposed "state-relevance coefficient" should help to choose which states to consider in a reduced model in each study case. Detailed nonlinear simulation results show that the proposed algorithm is able to find the relevant states to include in the reduced model systematically, even in operation points near the stability limit, where ad-hoc MOR techniques are likely to fail. The performance of the algorithm is illustrated in a system with grid-forming converters in various scenarios but can be easily applied to other systems.
\end{abstract}

Index Terms-DC-AC power conversion, distributed control, dynamics, reduced order systems, state space methods.

\section{INTRODUCTION}

$\mathbf{M}$ ICROGRIDS are considered fundamental tools for the penetration of renewable energy resources (RERs) by means of distributed generation (DG). Microgrids can operate in interconnected or in islanded mode. Since DG nearly always involves RERs, microgrids are populated by electronic converters, which make tight and fast control possible. Among those converters, only voltage-source converters (VSCs) are considered a viable alternative nowadays.

The control of islanded microgrids is usually organized hierarchically. It includes primary control, secondary control, and tertiary control [1]. Primary control seeks to stabilize the voltage and frequency across the microgrid after any change

Andrés Tomás-Martín, Aurelio García-Cerrada and Lukas Sigrist are with the Institute for Research in Technology, ICAI, Comillas Pontifical University, Madrid, Spain.

Sauro Yagüe is with the IQS Department of Industrial Engineering, (Ramón Llull University), Barcelona, Spain.

Jorge Suárez-Porras: Formerly with the Institute for Research in Technology, ICAI, Comillas Pontifical University. Now is a student of the Master's program: Power Electronics and Drives at AAU Energy at Aalborg University, Denmark.

This work has been partially financed through the research program S2018/EMT-4366 PROMINT-CAM on Smart Grids of Madrid Government, Spain with $50 \%$ support of the European Social Fund and by Grant RTI2018098865-B-C31 and by Grant RTC-2017-6296-3 funded by MCIN/AEI/ $10.13039 / 501100011033$ and by "ERDF A way of making Europe".

Corresponding author: Andrés Tomás-Martín. Email: atomas@comillas.edu or disturbance (e.g., either an islanding event or a change in generated or demanded power when operating in islanded mode, already). This control level is above the often-called level-0 in which converter output-current and/or output-voltage control are implemented. Level-0 determines if a VSC is a grid-forming or a grid-follower type. The former imposes the voltage and the frequency at the point of common coupling (PCC) and it can be called voltage-controlled voltage-source converter (VCVSC) while the latter injects a current orientated with respect to the voltage at the PCC and can be called current-controlled voltage-source converter (CCVSC). In both cases, primary control will elaborate the set-point signals for level-0 to satisfy specific objectives.

Secondary control restores frequency and voltage across the microgrid to their nominal values after a disturbance and imposes the most convenient voltage profile across the microgrid. Secondary control can be decentralized (without communications) like in [2], distributed (with communications only between some DGs) like in [3]-[5], and centralized (with all DGs communicating with a central controller) like in [6].

Tertiary control, which includes the energy management system, determines the optimal resource allocation and the active and reactive power or bus-voltage set points.

When analyzing or designing any of the above control levels, having a reduced-order model of lower levels is very convenient to prevent a soaring complexity. Model order reduction (MOR) has been widely applied to conventional, largescale power systems where transmission line dynamics and stator flux dynamics in synchronous generators are neglected due to the significant speed separation between them and synchronous generators' speed dynamics [7]. However, in microgrids with a high penetration of RERs, MOR algorithms may not benefit, a priori, from this well-established timescale separation. In fact, VCVSCs and CCVSCs can control their output variables, including frequency, very quickly (in a few $\mathrm{ms})$. In this scenario, many researchers propose slowing down frequency variations in converters $[\overline{8}]$ to simulate some form of inertia but it might not be desirable to go back to a conventional dynamic separation altogether, when most generation comes from electronic converters, due to a possible loss of flexibility.

Most MOR-related literature in low inertia microgrids apply conventional methods (e.g., neglecting line dynamics and simplifying level-0 of converter control by considering either current control or current+voltage control instantaneous [9]). 
These ad-hoc simplifications are then validated comparing the response of the complete (often nonlinear) and simplified systems under very specific assumptions at a limited number of operating points [10]. The small number of more systematic approaches applied to microgrids (e.g., those that take into account the importance of the system modes in its input-output response) is the main motivation of this paper. Systematic approaches of MOR use techniques that, among others, include state-space transformation, moment matching, and singular perturbation [11].

State-space transformation techniques include balanced truncation and the Hankel-norm method. Balanced truncation uses a "balanced" realization of the original system which is obtained through a linear transformation. The energy in the system output related to each of the states of the new realization is quantified by the Hankel singular values (HSVs) and the states with small HSVs can be left out of the system description (truncated) [12]. This method has been used for MOR in power systems in [13], resulting in a much lower order reduced system. However, the physical meaning of the system states is not preserved and therefore the original statesdynamics relation is lost. The Hankel-norm method minimizes the error bound (sum of the HSVs of the neglected states) of the transformation [11], [14].

Moment-matching techniques (also known as Krylov-based techniques) are transfer-function-based methods, whose objective is to match the behavior of the first terms of the power series expansions of the system. A moment-matching method commonly used to approximate communication delays is Padé's approximation [11], [15].

Common to most MOR algorithms included above is the use of a state-variable transformation which leads to a reducedorder model where the new system states do not preserve the physical meaning of the original ones.

Singular perturbation techniques are also popular in MOR [16] and have been applied to microgrids before [17], [18]. Although the last two references spell out the procedure to reduce the model once the relevant state variables are known, they do not explain this selection systematically. Furthermore, they only look at the speed of the state dynamics to make the selection.

This paper presents a systematic MOR algorithm to extract the relevant physical states of a linear system with application to microgrids with electronic power sources. Although the case study presented uses VCVSC with droop control, the method could be applied to other converter types and control strategies. The proposed algorithm uses a balanced realization of the system to calculate the energy contribution of each state to the output of the transformed system. It then uses that state energy and the participation factors of the linear system eigenvalues in the states of the original and transformed systems to calculate the relevance of each state in the original system. One must include these relevant states in reduced models to preserve a similar input-output and input-state response.

The paper is organized as follows: Section [II explains the proposed algorithm, Section II-A introduces the concept of balanced realization of a linear system. Sections II-B and II-C spell out the algorithms to calculate the eigenvalue relevance and the state relevance, respectively. Section III applies the proposed MOR algorithm to a small microgrid to understand the behavior of the system. The results obtained when applying the idea of state relevance are discussed in Section [V] and Section $\mathrm{V}$ concludes the paper.

\section{THE MODEL ORDER REDUCTION ALGORITHM}

MOR in any linear system involves:

1) Identification of relevant dynamics.

2) Identification of the states participated by those relevant dynamics.

3) Reduction of the model to include the dynamics of the relevant states plus the algebraic equations to calculate the non-relevant states.

\section{A. Balanced state-space realization of a linear system}

Let us consider a linear system with the form:

$$
\left\{\begin{array}{l}
\dot{x}=\mathbf{A} x+\mathbf{B} u \\
y=\mathbf{C} x+\mathbf{D} u
\end{array}\right.
$$

where $x$ is the system state column vector, $u$ is the input vector, $y$ is the output vector and $\mathbf{A}, \mathbf{B}, \mathbf{C}$ and $\mathbf{D}$ are the system matrices.

If the system in (1) is asymptotically stable, the controllability $\left(\mathbf{W}_{c}^{2}\right)$ and observability $\left(\mathbf{W}_{o}^{2}\right)$ Gramians are defined in [19] as:

$$
\left\{\begin{array}{r}
\mathbf{W}_{c}^{2} \triangleq \int_{0}^{\infty} e^{\mathbf{A} t} \mathbf{B B}^{\mathrm{T}} e^{\mathbf{A}^{\mathrm{T}} t} \mathrm{~d} t \\
\mathbf{W}_{o}^{2} \triangleq \int_{0}^{\infty} e^{\mathbf{A}^{\mathrm{T}} t} \mathbf{C}^{\mathrm{T}} \mathbf{C} e^{\mathbf{A} t} \mathrm{~d} t
\end{array}\right.
$$

The linear system can be transformed into another one whose states $\bar{x}$ are a linear combination of the states of the original system $(x)$ given by a transformation matrix $\mathbf{T}$ such that $\bar{x}=\mathbf{T} x$ and $x=\mathbf{T}^{-1} \bar{x}[12]$. The linear system is consequently transformed into:

$$
\left\{\begin{array}{l}
\dot{\bar{x}}=\mathbf{T A T}^{-1} \bar{x}+\mathbf{T B} u \\
y=\mathbf{C T}^{-1} \bar{x}+\mathbf{D} u
\end{array}\right.
$$

and the controllability and observability Gramians are:

$$
\overline{\mathbf{W}}_{c}^{2}=\mathbf{T} \mathbf{W}_{c}^{2} \mathbf{T}^{T} \quad \overline{\mathbf{W}}_{o}^{2}=\left(\mathbf{T}^{-1}\right)^{T} \mathbf{W}_{o}^{2} \mathbf{T}^{-1}
$$

A balanced transformation meets:

$$
\overline{\mathbf{W}}_{c}^{2}=\overline{\mathbf{W}}_{o}^{2}=\operatorname{diag}\left(g_{i}\right)
$$

where $g_{i}$ are the HSVs. Small entries in $g_{i}$ indicate states that can be removed to simplify the model while large entries indicate the most relevant states [19]. 


\section{B. Eigenvalue relevance analysis}

Since the balanced transformation is linear (each new state is a linear combination of the original states), the transformed system has the same eigenvalues (modes) as the original system. Therefore, relevant eigenvalues of the transformed system will also be relevant eigenvalues of the original system. Relevant states can be chosen based on the values of $g_{i}$ after the transformation defined in (3)-(5) and relevant eigenvalues can be determined checking which eigenvalues have high participation factors in the relevant states. The mode-in-state participation factors normalized as in [20] (based on [21]) are used here:

$$
p_{j i}=\frac{\left|w_{i j}\right|\left|v_{j i}\right|}{\sum_{\forall k}\left|w_{i k}\right|\left|v_{k i}\right|}
$$

where $p_{j i}$ is the normalized mode(i)-in-state( $\mathrm{j}$ ) participation factor, $v_{j i}$ is the element of the $j-t h$ row and $i-t h$ column of matrix $\mathbf{V}$ of right column eigenvectors and $w_{i j}$ is the element of the $i-t h$ row and $j-t h$ column of matrix $\mathbf{W}$ of left row eigenvectors calculated as $\mathbf{W}=\mathbf{V}^{-1}$. Notice that while $v_{j i}$ and $w_{i j}$ are general complex numbers, $p_{i j}$ is always a real number greater than 0 .

However, since each state of the transformed system has a different relevance, it is proposed that the participation factor of each eigenvalue in the states of the transformed system should be weighted with the state relevance (i.e., an eigenvalue having a high participation factor in a relevant state is not necessarily more relevant than an eigenvalue having a low participation factor in a more relevant state). The value of $g_{j}$ can then be used to weight $\bar{p}_{i j}$ using:

$$
\hat{\mathbf{R}}_{\lambda}=\left[\hat{R}_{\lambda}\left(\lambda_{1}\right), \cdots, \hat{R}_{\lambda}\left(\lambda_{n}\right)\right]^{T}=\left(\left[g_{1}, \cdots, g_{n}\right] \cdot \overline{\mathbf{P}}\right)^{T}
$$

where $\hat{R}\left(\lambda_{i}\right)$ will be called the "relevance of eigenvalue $\lambda_{i}$ " and $\overline{\mathbf{P}}$ is the participation matrix of the transformed system which has $\bar{p}_{i j}$ in its $i-t h$ row and $j-t h$ column. Normalizing, yields:

$$
\mathbf{R}_{\lambda}=\left[R_{\lambda}\left(\lambda_{1}\right), \ldots, R_{\lambda}\left(\lambda_{n}\right)\right]^{T}=\hat{\mathbf{R}}_{\lambda} / \max \left(\hat{\mathbf{R}}_{\lambda}\right)
$$

The normalized value will be used unless otherwise stated.

\section{State relevance}

Let us weight each column of the participation matrix of the original system $(\mathbf{P})$ with the relevance of its associated eigenvalue $\left(\hat{R}\left(\lambda_{i}\right)\right)$. By summing all these weighted columns, the resulting column vector can be used to quantify the "relevance of each state":

$$
\hat{\mathbf{R}}_{x}=\mathbf{P} \cdot \mathbf{R}_{\lambda}
$$

which can be normalized as follows:

$\mathbf{R}_{x}=\left[R_{x}\left(x_{1}\right), \ldots, R_{x}\left(x_{n}\right)\right]^{T}=\hat{\mathbf{R}}_{x} / \sum_{\forall i}\left(\hat{R}_{x}\left(x_{i}\right)\right)$

where $R_{x}\left(x_{i}\right)$ will be called the "relevance coefficient of state $x_{i}$ ". The normalized value will be used unless otherwise stated.

\section{Application to A CASE Study: CONTROL OF A MICROGRID WITH TWO VCVSCS}

\section{A. Description of the case study}

The MOR technique described before has been applied to a simple microgrid consisting of two VCVSCs (DG1 and DG2) connected to two buses where two loads (load 1 and load2) are also connected. The two buses are connected by a power line (see Fig. 1). The parameters of the line $\left(R_{\text {line }}\right.$ and $\left.L_{\text {line }}\right)$ and loads in Fig. 1 are given in Table I]

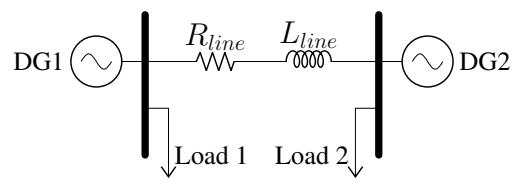

Fig. 1. Microgrid with two VCVSCs and two loads

TABLE I

PARAMETERS USED FOR THE SIMULATION OF THE MICROGRID WITH 2 VCVSCS

\begin{tabular}{lll}
\hline VCVSCs & DG 1 & DG 2 \\
\hline$m_{P i}$ & $1.30 \cdot 10^{-3} \mathrm{~V} / \mathrm{W}$ & $1.50 \cdot 10^{-3} \mathrm{~V} / \mathrm{W}$ \\
$n_{Q i}$ & $9.40 \cdot 10^{-5} \mathrm{rad} / \mathrm{s} \cdot \mathrm{VAr}$ & $1.25 \cdot 10^{-4} \mathrm{rad} / \mathrm{s} \cdot \mathrm{VAr}$ \\
$R_{f i}$ & $0.1 \Omega$ & $0.1 \Omega$ \\
$L_{f i}$ & $1.35 \mathrm{mH}$ & $1.35 \mathrm{mH}$ \\
$C_{f i}$ & $50 \mu \mathrm{F}$ & $50 \mu \mathrm{F}$ \\
$K_{P V i}$ & 0.1 & 0.05 \\
$K_{I V i}$ & 420 & 390 \\
$K_{P C i}$ & 15 & 10.5 \\
$K_{I C i}$ & 20000 & 16000 \\
$F_{i}$ & 0.75 & 0.75 \\
$L P F_{P}$ & $0.05 \mathrm{~s}$ & $0.05 \mathrm{~s}$ \\
$L_{P} F_{Q}$ & $0.01 \mathrm{~s}$ & $0.01 \mathrm{~s}$ \\
\hline Sec. control, bases & & \\
\hline$c_{f}, c_{v}$ & 10 & 10 \\
$f_{\text {base }}, S_{\text {base }}$ & $50 \mathrm{~Hz}$ & $10 \mathrm{kVA}$ \\
delay, $V_{\text {nom }}=V_{\text {base }}$ & $1.00 \cdot 10^{-5} \mathrm{~s}$ & $380 \mathrm{~V}$ \\
{$[\beta, B], Z_{\text {base }}$} & {$[0,1]$} & $43.32 \Omega$ \\
\hline Line & & \\
\hline$R_{\text {line }}$ & $1 \Omega$ & 2 \\
$L_{\text {line }}$ & $1 \mathrm{mH}$ & 0.064 \\
\hline Loads & $\mathbf{1}$ & \\
\hline$R_{L i}(\Omega)$ & 30 & \\
$X_{L i}(\Omega)$ & 15.0796 & \\
$L_{L i}(\mathrm{H})$ & 0.048 & \\
\hline & & \\
\hline
\end{tabular}

The schematic of each VCVSC is shown in Fig. 2. It is connected to the bus or PCC and imposes the voltage and frequency on its point of control (PC). For several important reasons, this type of electronic converter usually provides an inner current controller. Typically, PI voltage and current controllers in a synchronously rotating frame $(d-q)$ guarantee that voltage and current follow their set-points values [5].

The $a b c / d q$ block in Fig. 2 is the so-called Park's Transform, which converts the 3-phase voltage (or current) signal to its direct-quadrature $(d-q)$ "components" in a reference frame that rotates with the frequency of the 3-phase signal which is imposed by the VCVSC. In this reference frame, $d-q$ 


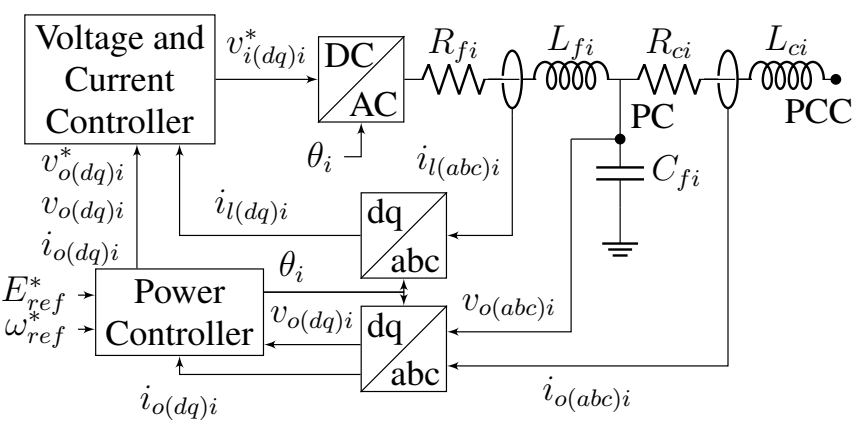

Fig. 2. Schematics of a VCVSC. The abc/dq block is the so-called Park Transform. Adapted from [5]

components of all electrical variables are constants in steadystate in balanced systems and simple PI controllers for power control, voltage control, or current control are effective. Each VCVSC uses its reference frame for the Park's transform for control purposes such that the set point for its $q$ component is zero $\left(v_{o q i}^{*}=0\right)$. Applying a power-invariant Park's Transform, active and reactive power drawn from a VCVSC have a similar expression to the one using $a b c$ electrical variables. For simplicity, the output inductances of both VCVSCs $\left(L_{c 1}\right.$ and $L_{c 2}$ ) are considered together with the actual transmissionline inductance $\left(L_{\text {line }}^{*}\right)$ inside the transmission-line variable $\left(L_{\text {line }}=L_{c 1}+L_{c 2}+L_{\text {line }}^{*}\right.$, and the same for $R$ ). For modeling and simulation purposes of the whole system, one of the $d-q$ synchronous reference systems is taken as angle reference and all other state variables are referred to it. In this paper, DG1 is that reference.

The simplest model of a VCVSC is an ideal voltage source that imposes the voltage and frequency at its point of control. Including the voltage and current controllers makes the model more accurate, which is in general necessary for stability studies related to converter interactions. Typically, the converter itself, presented by the DC/AC conversion block in Fig. 2, is modeled as a controlled ideal sinusoidal voltage source by neglecting the PWM-driven switching of the switches (e.g., IGBTs). Switching transients are much faster than the voltage and current controller's dynamics. Given the rather fast controller dynamics, line dynamics of the microgrid cannot be neglected a priori. Obviously, the presence of several VCVSCs and other elements together with the inclusion of line dynamics significantly increases the size of the problem. Besides if, for instance, during a black-start process, several microgrids were coordinated, the problem size would increase further. MOR aims at simplifying models without significantly sacrificing their accuracy. Clearly, MOR is application-dependent, and the focus is here on the stability of a microgrid.

A typical control level-0 (i.e., output current and voltage controllers) of a VCVSC [4] is shown in Fig. 3

If more than one VCVSC must work in parallel, some form of droop control must be provided. Figure 4 illustrates the $\omega-Q$ and $v-P$ droop controller of a VCVSC used in this case study where the actual active and reactive powers delivered by each converter $i\left(P_{i}\right.$ and $Q_{i}$, respectively) are calculated from

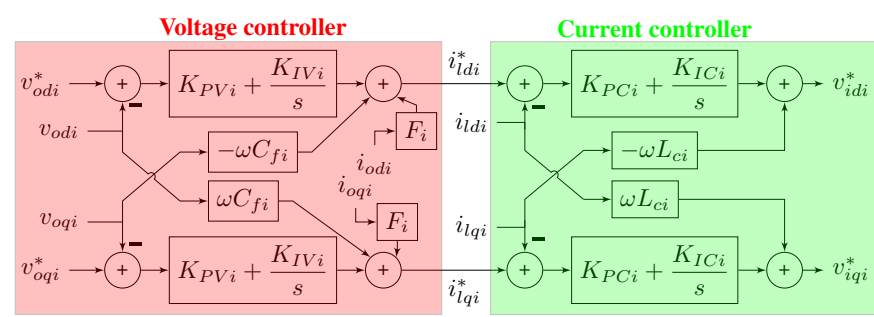

Fig. 3. Voltage and current controllers of a VCVSC, including the decoupling of the output LC filter and current feed-forward. Adapted from [4]

its output voltage and current. The results are filtered by a low-pass filter and used to manipulate the output voltage and frequency set-point values. Notice that typical $\omega-P$ and $v-Q$ droop controllers have not been used because a low-voltage line with a low $\mathrm{X} / \mathrm{R}$ ratio connects the power converters. Control level-0 parameters and droop-controller parameters are also given in Table $\mathrm{I}$

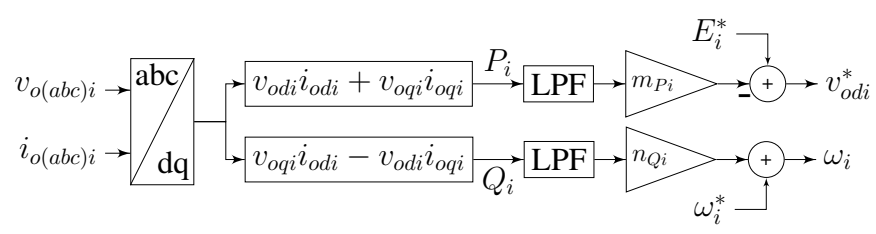

Fig. 4. Power controller of a VCVSC, involving the droop control methods for frequency and voltage. low-pass filters (LPFs) are included to reduce measurement noise

A secondary control layer has also been implemented in the system shown in Fig. 1. It takes the form of a multi-agentbased distributed secondary control. Unlike in a centralized controller, DGs must collaborate with each other to reach a solution, called "consensus value". Multi-agent-based control is attractive for microgrids given the dispersed nature of RERs. Ref. [22] includes more details on cooperative multiagent secondary control applied to microgrids, along with the conditions that the communication graph must meet to have a stable system.

Figures 5 and 6 show the multi-agent secondary control of each VCVSC used in this case study.

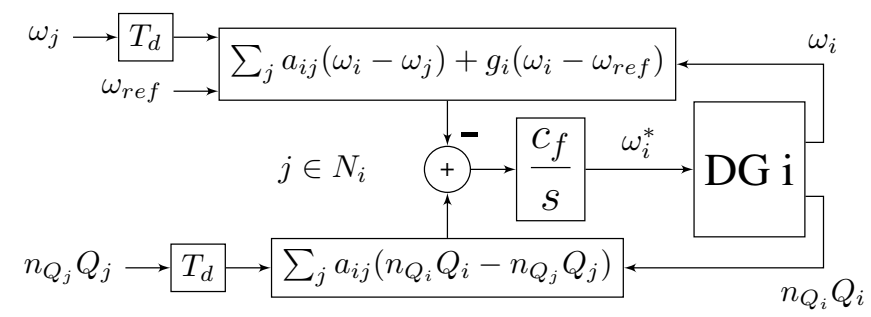

Fig. 5. Multi-agent secondary $\omega$-Q control of a VCVSC with $N_{i}$ neighbors. $T_{d}$ represents a constant communication time delay from DG $\mathrm{j}$ to DG $\mathrm{i}$

The secondary control structure in Figs. 5 and 6 is similar to the one included in [5] for the VCVSCs but it has been adapted to resistive $\mathrm{LV}$ lines, to guarantee active and reactive power sharing among both converters. However, VCVSCs will not seek voltage consensus ( $B=1 ; \beta=0$ ) to make consensus 


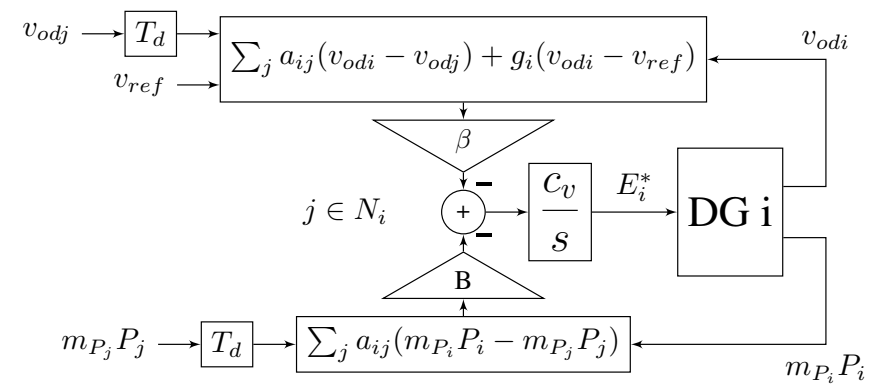

Fig. 6. Multi-agent secondary v-P control of a VCVSC

in active-power sharing possible. In microgrids with highly resistive transmission lines, the active-power transfer between two nodes mostly depends on the voltage difference between them. Therefore, voltage profile and active power flow cannot be controlled simultaneously (as explained in [3] for inductive lines and v-Q dependency). All parameters for Figs. 5 and 6 are given in Table I. The parameters of the test system are based on those in [4]. In the current case study, a simple graph is used in which DG1 is the leader, following $v_{\text {ref }}=\omega_{\text {ref }}=1$ p.u., and DG2 follows the frequency and active and reactive power sharing of DG1. A very small constant communication delay between the two converters is included to show that the state relevance finds it negligible. A thorough analysis of the effect of communication delays is out of the scope of this paper. Delays are modeled using a third-order Padé approximation in the linear model and an exact delay in the nonlinear model.

\section{RESUlts}

The system in Fig. 1 has been linearized by using MATLAB $\AA$ and Simulink ${ }^{\circledR}$ for two different cases to illustrate the use of the state-relevance coefficient for MOR. Line, loads, and controller dynamics were all included. Case 1 has the original parameters (see Table I), and case 2 explores the effect of changing the line impedance while keeping a constant line $L$ to- $R$ ratio. The impedance value in case 2 is chosen to bring the system close to its stability limit, a region in which MOR techniques are more likely to fail.

Figure 7 compares the state-relevance coefficient in Section II-C in logarithmic scale, when applied to case 1 (in blue diamonds) and case 2 (in red squares). See Tables $\Pi$ and $\Pi I I$ included in appendix $\mathrm{A}$ for the exact values of state relevance and the state order for each case. It has been considered that there was an important drop in the state-relevance coefficient when there was a difference of more than 0.7 between two successive values of $\log \left(R_{x}\left(x_{i}\right)\right)$. If no drop was found, the threshold for the logarithmic jump was reduced. To avoid detecting a premature jump, the most relevant state after the jump must have a relevance lower than 0.01, and the less relevant state before the jump must have a relevance higher than 0.001. The figure shows that in case 2 one has to consider more states before the first important drop in the state-relevance coefficient is detected. The color-filled state is the last relevant state recommended to be considered in the reduced model (state 9 in the former and state 15 in the latter).

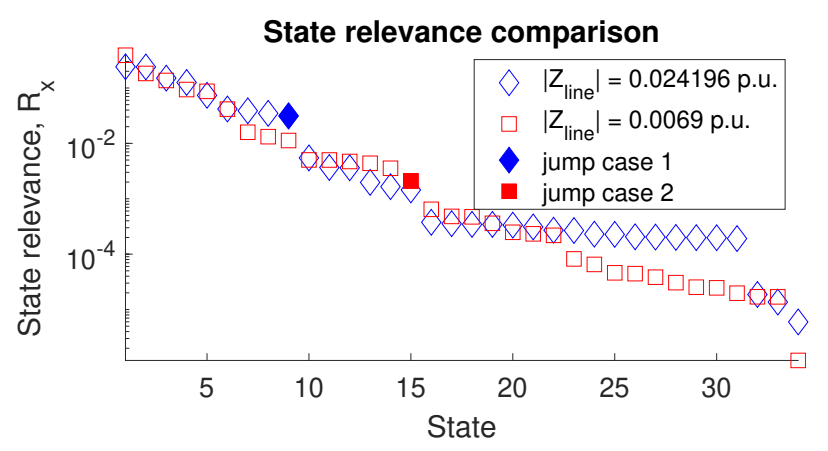

Fig. 7. State-relevance comparison in two case studies. Color-filled, the last relevant state before the drop in the state-relevance coefficient. For clarity, only states 1-34 are shown (see Tables II and III for complete data)

Figure 8 a shows the evolution of the relevance of the most relevant state belonging to the transmission when changing the line-impedance value while maintaining a constant $L$-to$R$ ratio (continuous blue line). In addition, the position of the transmission line state in the list of states sorted according to the relevance is shown (black line with circular markers) and compared with the position of the last relevant state (dashed red line). Clearly, the relevance of the transmission line states decreases as the impedance increases. In fact, when the line impedance is high enough, the transmission line states fall behind the last relevant one. Consequently, the transmissionline dynamics can be neglected if its impedance is high enough, whilst those dynamics must be considered if the line impedance is relatively small. This result agrees well with the intuitive idea of needing a relatively high impedance between two voltage sources (in this case two VCVSCs). Indeed, if the transmission line has a low impedance, any slight change in the voltage set by one VCVSC can drive a significant current change and lead the system to instability.

Figure $8 \mathrm{~b}$ shows the evolution of the relevance of the most relevant state of the transmission line when changing the line $L$-to- $R$ ratio while maintaining a constant impedance value. Again, the number of the last relevant state is marked with a dashed red line and the number of the most relevant of the line states is marked with a black line with circular markers. Clearly, the transmission line dynamics can be neglected if its $L$-to- $R$ ratio is low. However, if the $L$-to- $R$ ratio increases ( $L$ increases and $R$ decreases, the line time constant increases), the transmission line dynamics must be considered.

Figure 9 illustrates how the system modes participate in the states of the original system. Mode-in-state participations have been calculated using the normalization in [20] (based on [21]). Notice that a dark Grey color indicates those states with a large participation of a given mode. The axis at the bottom of Fig. 9 measures the inverse of the real parts of the system modes (i.e. the time constant of the decaying exponential of a stable mode). For example, modes 1 to 9 have an exponential time constant just above $10^{-6}$ seconds. The states of the time-delay blocks have the fastest dynamics, followed by the voltage- and current-controllers dynamics and the loads. The transmission-line dynamics are much slower but still faster than those of the LPF filter of the power controllers 


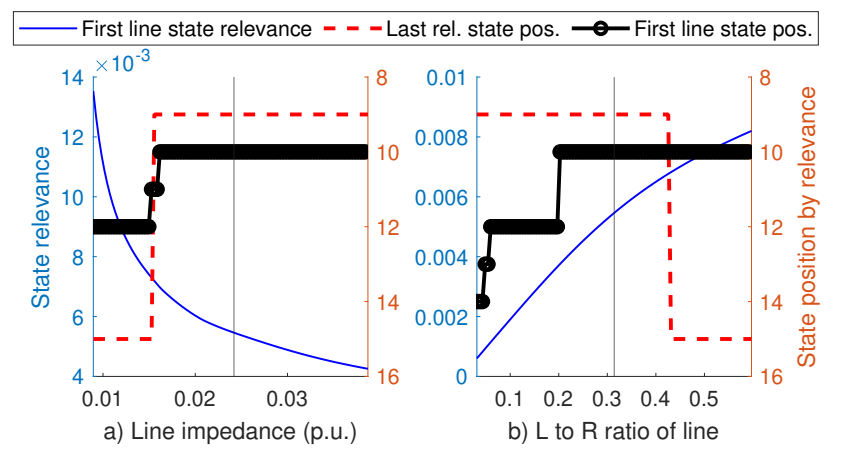

Fig. 8. Evolution of the relevance of the transmission line when changing its impedance and $L$ to $R$ ratio. The left vertical axis represents the relevance of the most relevant transmission line state, and the right vertical axis represents the state position in the list of the system states ordered by relevance

and the secondary control of both VCVSCs.

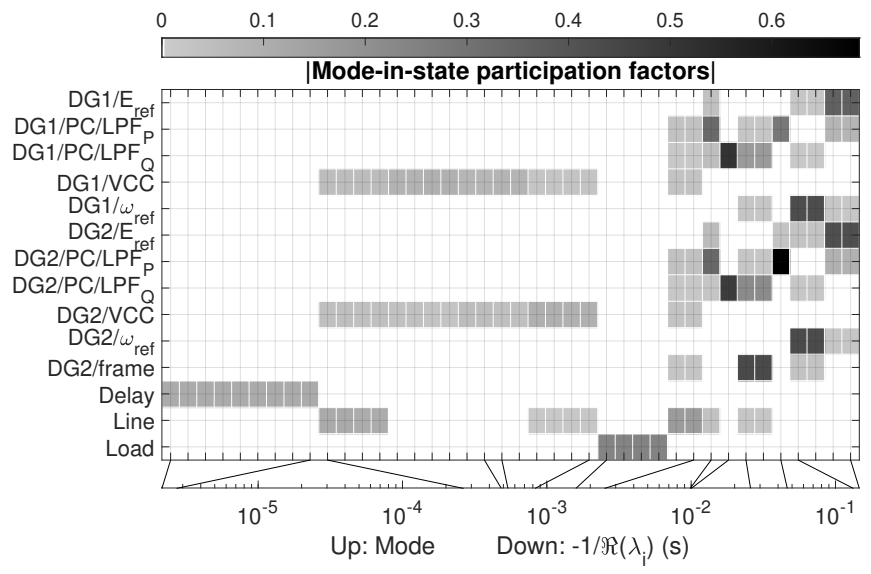

Fig. 9. Illustrating the participation factors of the eigenvalues in the states of the system. For clarity, participation factors below 0.01 are not shown States are grouped. The $\mathrm{x}$-axis also includes the time constant of the mode approximated as $-1 / \Re\left\{\lambda_{i}\right\}$ to show which states are participated by the fast or slow modes

Figure 10 shows the system-pole loci evolution when changing the transmission-line impedance while maintaining a constant $L$-to- $R$ ratio. A modulus vs angle representation has been used to show clearly poles with large and small modulus simultaneously (see caption for more details). Poles that move go from dark Grey to pale Grey as the line impedance decreases. Since the pole damping can be calculated from its angle, Figure 10 shows that several pole damping coefficients deteriorate when the transmission line impedance is reduced. Eventually, the system becomes unstable if that impedance becomes sufficiently small.

\section{A. Applying model reduction to the nonlinear model}

This section will investigate whether the state-relevance coefficient is helpful in the MOR of a nonlinear system of a microgrid. Several reduced models of the system in Fig. 1 will be compared by nonlinear simulation. Two approaches have been followed in order to obtain those reduced models: (a) the states participated by a selection of fast modes have been eliminated and (b) the state-relevance coefficient has been

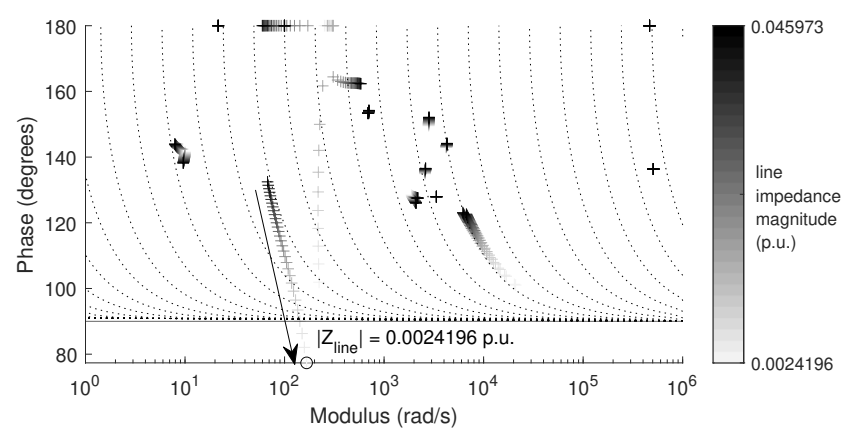

Fig. 10. Pole loci evolution when changing the transmission line impedance keeping a constant $L$ to $R$ ratio. Poles with a phase between $90^{\circ}$ and $270^{\circ}$ are stable. Only poles with phases up to $180^{\circ}$ are included in the graph omitting their complex conjugates. Dotted lines are constant real part lines.

used to decide which states should be eliminated. The fastest dynamics in an electrical microgrid may include, transmission lines, and voltage and current controllers. Appendix B includes the details of how the selected dynamics have been eliminated from the model in the following cases.

The original system's pole loci and time response with all dynamics considered (denoted as "Complete") are compared to three reduced systems for the two study cases considered previously. For the time response, a $20 \%$ reduction of the impedance of Load 1 (tantamount to increasing Load 1) will be simulated.

The first reduced system used ("Relevant") includes the dynamics for the most relevant states before an important drop in the state-relevance coefficient is detected. Therefore, states with neglected dynamics include output-filter capacitors, filter inductances, $\left(C_{f i}\right.$ and $L_{f i}$, respectively in Fig. 2), voltage and current controllers, loads, delays, and transmission line for case 1 , and current controller, output-filter capacitors, filter inductances, loads, and delays for case 2.

The second reduced system used ("SLinesVCC") includes a static transmission line and ideal VCVSCs, neglecting the dynamics of delays, output-filter capacitors, filter inductances, transmission line, loads, and voltage and current controllers.

The third reduced system used ('SLines") neglects the dynamics of delays, output-filter capacitors, filter inductances, transmission line, and loads.

Figure 11 shows the pole loci, and the time-response of DG2 frequency to a step increment in Load 1, of the original and reduced systems for case 1 . Figure 12 shows the same experiment for case 2 .

In case 1 , the reduced systems do not include the states with the fastest dynamics, and therefore their pole loci do not include poles with a very large module. All reduced models include those eigenvalues with slow time constants and they all have a time response very similar to the original model ("Complete").

In case 2 , the system has a very poorly damped eigenvalue (phase near $90^{\circ}$ ) with modulus near $10^{2} \mathrm{rad} / \mathrm{s}$. Only the socalled "Relevant" model reduction is able to include these poorly damped dynamics. Conventional "ad hoc" approaches fail to include this information. 

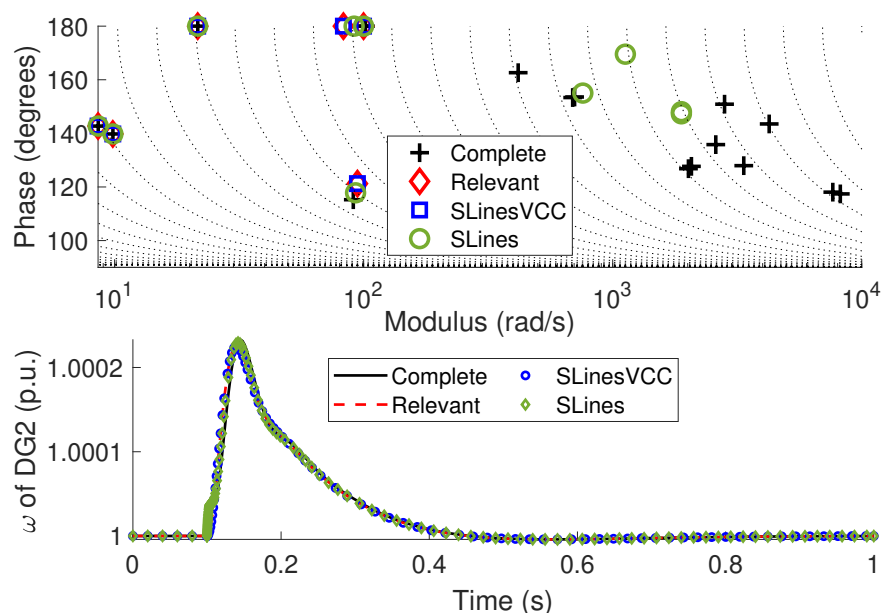

Fig. 11. Pole loci and time response to a change in Load 1 of the origina and reduced systems for case 1
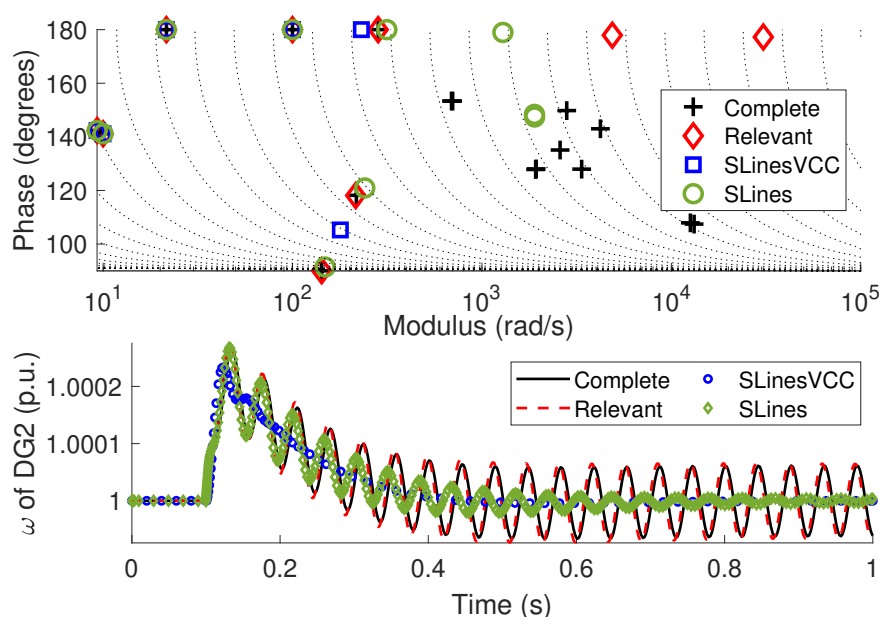

Fig. 12. Pole loci and time response to a change in Load 1 of the origina and reduced systems for case 2

\section{CONClusions}

This paper has proposed an algorithm to quantify the state relevance of a linear system while the physical meaning of the state variables is preserved and has described its application to a microgrid with electronic generation. The algorithm uses a balanced realization of the system to calculate the state energy in a different state space. Then, it calculates the state-relevance coefficient for the original system using that state energy and the original and transformed systems participation factors. The proposed MOR algorithm has been applied to a simple test system, and two case studies have been analyzed. The proposed algorithm has also been compared with conventional MOR techniques that calculate those states participated by fast system modes using algebraic equations. It can be concluded that if the system is far from its stability limits, the proposed and conventional MOR techniques produce equally accurate results. However, if the system is close to stability limits, conventional MOR techniques fail to do so. The proposed state-relevance coefficient can be used to choose the states to include in MORs for both study cases, systematically.
APPENDIX A

STATE-RELEVANCE TABLES

See Tables $\coprod$ and

TABLE II

STATE RELEVANCE OF THE TEST SYSTEM WITH LINE IMPEDANCE $=$ 0.024196 P.U.

\begin{tabular}{|c|c|c|c|c|c|}
\hline $\mathbf{n}$ & State name & $R_{x}$ & $\mathbf{n}$ & State name & $R_{x}$ \\
\hline 1 & $\mathrm{DG} 2 / \omega_{r e f}$ & 0.24175 & 21 & Load $1 / i_{q}$ & 0.00030988 \\
\hline 2 & $\mathrm{DG} 1 / \omega_{r e f}$ & 0.23967 & 22 & $\mathrm{DG} 1 / \mathrm{VCC} / \mathrm{RL} / i_{d}$ & 0.00027022 \\
\hline 3 & $\mathrm{DG} 2 / E_{r e f}$ & 0.15053 & 23 & $\mathrm{DG} 1 / \mathrm{VCC} / \mathrm{RL} / i_{q}$ & 0.00026713 \\
\hline 4 & $\mathrm{DG} 1 / E_{\text {ref }}$ & 0.12566 & 24 & $\mathrm{DG} 2 / \mathrm{VCC} / \mathrm{RL} / i_{d}$ & 0.00022944 \\
\hline 5 & DG2/frame & 0.073712 & 25 & $\mathrm{DG} 2 / \mathrm{VCC} / \mathrm{RL} / i_{q}$ & 0.00022746 \\
\hline 6 & $\mathrm{DG}^{2} / \mathrm{PC} / \mathrm{LPF}_{P}$ & 0.041751 & 26 & $\mathrm{DG} 1 / \mathrm{VCC} / \mathrm{PI} i_{d}$ & 0.00020576 \\
\hline 7 & $\mathrm{DG} / \mathrm{PC} / \mathrm{LPF}_{Q}$ & 0.038709 & 27 & $\mathrm{DG} 2 / \mathrm{VCC} / \mathrm{PI} i_{d}$ & 0.00020322 \\
\hline 8 & $\mathrm{DG} / \mathrm{PC} / \mathrm{LPF}_{P}$ & 0.034806 & 28 & $\mathrm{DG} 1 / \mathrm{VCC} / \mathrm{PI} i_{q}$ & 0.00020058 \\
\hline 9 & $\mathrm{DG} 1 / \mathrm{PC} / \mathrm{LPF}_{Q}$ & 0.031297 & 29 & $\mathrm{DG} 2 / \mathrm{VCC} / \mathrm{RC} / v_{d}$ & 0.00019881 \\
\hline 10 & Line $/ i_{q}$ & 0.0054611 & 30 & DG2/VCC/PI $i_{q}$ & 0.00019789 \\
\hline 11 & $\mathrm{DG} 2 / \mathrm{VCC} / \mathrm{PI} v_{q}$ & 0.0036676 & 31 & $\mathrm{DG} 2 / \mathrm{VCC} / \mathrm{RC} / v_{q}$ & 0.00019106 \\
\hline 12 & $\mathrm{DG} 1 / \mathrm{VCC} / \mathrm{PI} v_{q}$ & 0.0036205 & 32 & DG2/Delay $_{\omega}(3)$ & $1.8681 \mathrm{e}-05$ \\
\hline 13 & Line $/ i_{d}$ & 0.0019726 & 33 & DG2/Delay $_{Q}(3)$ & $1.3638 \mathrm{e}-05$ \\
\hline 14 & $\mathrm{DG} 1 / \mathrm{VCC} / \mathrm{PI} v_{d}$ & 0.001652 & 34 & DG2/Delay $_{P}(3)$ & $5.9739 \mathrm{e}-06$ \\
\hline 15 & $\mathrm{DG} 2 / \mathrm{VCC} / \mathrm{PI} v_{d}$ & 0.0014445 & 35 & DG2/Delay $_{\omega}(2)$ & $1.7695 \mathrm{e}-10$ \\
\hline 16 & Load $2 / i_{q}$ & 0.00037594 & 36 & DG2/Delay $_{P}(2)$ & $1.1539 \mathrm{e}-10$ \\
\hline 17 & $\mathrm{DG} 1 / \mathrm{VCC} / \mathrm{RC} / v_{d}$ & 0.00035481 & 37 & DG2/Delay $_{\omega}(1)$ & $1.0115 \mathrm{e}-10$ \\
\hline 18 & $\mathrm{DG} 1 / \mathrm{VCC} / \mathrm{RC} / v_{q}$ & 0.00034466 & 38 & DG2/Delay $_{P}(1)$ & $7.2185 \mathrm{e}-11$ \\
\hline 19 & Load $2 / i_{d}$ & 0.00034395 & 39 & DG2/Delay $_{Q}(2)$ & $2.5576 \mathrm{e}-11$ \\
\hline 20 & Load $1 / i_{d}$ & 0.00033377 & 40 & DG2/Delay $_{Q}(1)$ & $1.7281 \mathrm{e}-11$ \\
\hline
\end{tabular}

TABLE III

STATE RELEVANCE OF THE TEST SYSTEM WITH LINE IMPEDANCE $=$ 0.0069 P.U.

\begin{tabular}{|c|c|c|c|c|c|}
\hline $\mathbf{n}$ & State name & $R_{x}$ & $\mathbf{n}$ & State name & $R_{x}$ \\
\hline 1 & DG2/frame & 0.38994 & 21 & $\operatorname{Load} 2 / i_{q}$ & 0.00023257 \\
\hline 2 & $\mathrm{DG} 2 / \mathrm{PC} / \mathrm{LPF}_{Q}$ & 0.18301 & 22 & $\mathrm{DG} 1 / \mathrm{VCC} / \mathrm{RC} / v_{q}$ & 0.0002187 \\
\hline 3 & 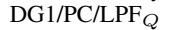 & 0.13669 & 23 & Load $1 / i_{q}$ & $8.2046 \mathrm{e}-05$ \\
\hline 4 & $\mathrm{DG} 2 / \mathrm{VCC} / \mathrm{PI} v_{q}$ & 0.093708 & 24 & $\mathrm{DG} 2 / \mathrm{VCC} / \mathrm{PI} i_{d}$ & $6.5245 \mathrm{e}-05$ \\
\hline 5 & $\mathrm{DG} 1 / \mathrm{VCC} / \mathrm{PI} v_{q}$ & 0.086969 & 25 & $\operatorname{Load} 2 / i_{d}$ & $4.603 \mathrm{e}-05$ \\
\hline 6 & Line $/ i_{q}$ & 0.041522 & 26 & $\mathrm{DG} 1 / \mathrm{VCC} / \mathrm{PI} i_{d}$ & $4.4471 \mathrm{e}-05$ \\
\hline 7 & $\mathrm{DG} 1 / \omega_{r e f}$ & 0.015877 & 27 & $\mathrm{DG} 2 / \mathrm{VCC} / \mathrm{RL} / i_{d}$ & $3.8373 \mathrm{e}-05$ \\
\hline 8 & $\mathrm{DG} / \mathrm{PC} / \mathrm{LPF}_{P}$ & 0.01321 & 28 & $\mathrm{DG} 1 / \mathrm{VCC} / \mathrm{RL} / i_{d}$ & $3.0586 \mathrm{e}-05$ \\
\hline 9 & 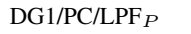 & 0.011259 & 29 & $\mathrm{DG} 2 / \mathrm{VCC} / \mathrm{RC} / v_{d}$ & $2.5322 \mathrm{e}-05$ \\
\hline 10 & $\mathrm{DG} 2 / E_{\text {ref }}$ & 0.005022 & 30 & $\mathrm{DG} 1 / \mathrm{VCC} / \mathrm{RC} / v_{d}$ & $2.4635 \mathrm{e}-05$ \\
\hline 11 & $\mathrm{DG} 2 / \omega_{\text {ref }}$ & 0.0050079 & 31 & $\operatorname{Load} 1 / i_{d}$ & $1.9723 \mathrm{e}-05$ \\
\hline 12 & $\mathrm{DG} 2 / \mathrm{VCC} / \mathrm{PI} v_{d}$ & 0.0047098 & 32 & DG2/Delay $_{\omega}$ (3) & $1.6994 \mathrm{e}-05$ \\
\hline 13 & $\mathrm{DG} 1 / \mathrm{VCC} / \mathrm{PI} v_{d}$ & 0.0043679 & 33 & DG2/Delay $_{Q}(3)$ & $1.6942 \mathrm{e}-05$ \\
\hline 14 & $\mathrm{DG} 1 / E_{\text {ref }}$ & 0.0035524 & 34 & DG2/Delay $_{P}(3)$ & $1.2056 \mathrm{e}-06$ \\
\hline 15 & Line $/ i_{d}$ & 0.0020949 & 35 & DG2/Delay $_{\omega}(2)$ & $6.3938 \mathrm{e}-12$ \\
\hline 16 & $\mathrm{DG} 2 / \mathrm{VCC} / \mathrm{RL} / i_{q}$ & 0.00064451 & 36 & DG2/Delay $_{Q}(2)$ & $5.3885 \mathrm{e}-12$ \\
\hline 17 & $\mathrm{DG} 1 / \mathrm{VCC} / \mathrm{RL} / i_{q}$ & 0.00048015 & 37 & DG2/Delay $_{P}(2)$ & $3.6069 \mathrm{e}-12$ \\
\hline 18 & $\mathrm{DG} 2 / \mathrm{VCC} / \mathrm{PI} i_{q}$ & 0.00047161 & 38 & DG2/Delay $_{\omega}(1)$ & $2.5483 \mathrm{e}-12$ \\
\hline 19 & $\mathrm{DG} 1 / \mathrm{VCC} / \mathrm{PI} i_{q}$ & 0.00036024 & 39 & DG2/Delay $_{P}(1)$ & $2.1959 \mathrm{e}-12$ \\
\hline 20 & $\mathrm{DG} 2 / \mathrm{VCC} / \mathrm{RC} / v_{q}$ & 0.00024818 & 40 & DG2/Delay $_{Q}(1)$ & $2.0925 \mathrm{e}-12$ \\
\hline
\end{tabular}

\section{APPENDIX B}

REDUCING STATES IN THE NONLINEAR MODEL

\section{A. Electrical elements with no dynamics}

The equations of a balanced inductance with series $R$ in a $d-q$ reference frame are:

$$
\left\{\begin{array}{c}
v_{d}=L \frac{d i_{d}}{d t}-\omega L i_{q}+R i_{d} \\
v_{q}=L \frac{d i_{q}}{d t}+\omega L i_{d}+R i_{q}
\end{array}\right.
$$

If the inductance dynamics are considered very fast, $d i_{d q} / d t=0$ very quickly and: 


$$
\left[\begin{array}{c}
i_{d} \\
i_{q}
\end{array}\right]=\left[\begin{array}{cc}
R & -\omega L \\
\omega L & R
\end{array}\right]^{-1}\left[\begin{array}{c}
v_{d} \\
v_{q}
\end{array}\right]
$$

Similarly, the equations for a ideal and balanced capacitor with parallel $R$ in a $d-q$ frame are:

$$
\left\{\begin{array}{c}
i_{d}=C \frac{d v_{d}}{d t}-\omega C v_{q}+\frac{1}{R} v_{d} \\
i_{q}=C \frac{d v_{q}}{d t}+\omega C v_{d}+\frac{1}{R} v_{q}
\end{array}\right.
$$

Making $d v_{d q} / d t=0$ yields:

$$
\left[\begin{array}{l}
v_{d} \\
v_{q}
\end{array}\right]=\left[\begin{array}{cc}
1 / R & -\omega C \\
\omega C & 1 / R
\end{array}\right]^{-1}\left[\begin{array}{l}
i_{d} \\
i_{q}
\end{array}\right]
$$

\section{B. Voltage and current controllers}

If the current controller dynamics are not relevant, but the voltage controller dynamics are relevant, the VSC model has been simplified by assuming that the converter output currents follow their references $(*)$ instantaneously:

$$
i_{l d i}^{*}=i_{l d i} \& i_{l q i}^{*}=i_{l q i}
$$

In addition, if the dynamics of the voltage controllers are not relevant, the VSC model can be simplified further as:

$$
v_{i d i}^{*}=v_{o d i} \& v_{i q i}^{*}=v_{o q i}
$$

\section{REFERENCES}

[1] A. Bidram and A. Davoudi, "Hierarchical Structure of Microgrids Control System," IEEE Trans. Smart Grid, vol. 3, no. 4, pp. 1963-1976, Dec. 2012

[2] Y. Khayat, R. Heydari, M. Naderi, T. Dragicevic, Q. Shafiee, M. Fathi, H. Bevrani, and F. Blaabjerg, "Estimation-based Consensus Approach for Decentralized Frequency Control of AC Microgrids," in 2019 21st European Conference on Power Electronics and Applications (EPE' 19 ECCE Europe), Sep. 2019, pp. 1-8.

[3] J. W. Simpson-Porco, Q. Shafiee, F. Dorfler, J. C. Vasquez, J. M. Guerrero, and F. Bullo, "Secondary Frequency and Voltage Control of Islanded Microgrids via Distributed Averaging," IEEE Trans. Ind. Electron., vol. 62, no. 11, pp. 7025-7038, Nov. 2015.

[4] A. Bidram, A. Davoudi, F. L. Lewis, and J. M. Guerrero, "Distributed Cooperative Secondary Control of Microgrids Using Feedback Linearization," IEEE Trans. Power Syst., vol. 28, no. 3, pp. 3462-3470, Aug. 2013.

[5] A. Bidram, A. Davoudi, and F. L. Lewis, "A Multiobjective Distributed Control Framework for Islanded AC Microgrids," IEEE Trans. Ind. Inf., vol. 10, no. 3, pp. 1785-1798, Aug. 2014.

[6] X. Wang, J. M. Guerrero, F. Blaabjerg, and Z. Chen, "Secondary voltage control for harmonics suppression in islanded microgrids," in 2011 IEEE Power and Energy Society General Meeting, Jul. 2011, pp. 1-8.

[7] P. Kundur, N. J. Balu, and M. G. Lauby, Power System Stability and Control, ser. The EPRI Power System Engineering Series. New York: McGraw-Hill, 1994.

[8] H. Bevrani and J. Raisch, "On Virtual inertia Application in Power Grid Frequency Control," Energy Procedia, vol. 141, pp. 681-688, Dec. 2017.

[9] T. Qoria, Q. Cossart, C. Li, X. Guillaud, F. Colas, F. Gruson, and X. Kestelyn, "WP3 - Control and Operation of a Grid with 100\% Converter-Based Devices. Deliverable 3.2: Local control and simulation tools for large transmission systems," MIGRATE project, Tech. Rep., Dec. 2018, accessed 25/11/2021. [Online]. Available: https://www.h2020-migrate.eu/downloads.html

[10] Y. Ojo, J. Watson, and I. Lestas, "A Review of Reduced-Order Mod els for Microgrids: Simplifications vs Accuracy," arXiv:2003.04923 [math.OC], Mar. 2020

[11] A. C. Antoulas, Approximation of Large-Scale Dynamical Systems. Philadelphia: Society for Industrial and Applied Mathematics, Jan. 2005.
[12] A. Laub, M. Heath, C. Paige, and R. Ward, "Computation of system balancing transformations and other applications of simultaneous diagonalization algorithms," IEEE Trans. Automat. Contr., vol. 32, no. 2, pp. 115-122, Feb. 1987

[13] A. Ramirez, A. Mehrizi-Sani, D. Hussein, M. Matar, M. Abdel-Rahman, J. Jesus Chavez, A. Davoudi, and S. Kamalasadan, "Application of Balanced Realizations for Model-Order Reduction of Dynamic Power System Equivalents," IEEE Trans. Power Delivery, vol. 31, no. 5, pp. 2304-2312, Oct. 2016

[14] A. Antoulas and D. Sorensen, "Approximation of large-scale dynamical systems: An overview," Int. J. Appl. Math. Comput. Sci., vol. 11, no. 5, pp. 1093-1121, 2001.

[15] R. Olfati-Saber and R. Murray, "Consensus Problems in Networks of Agents With Switching Topology and Time-Delays," IEEE Trans. Autom. Control, vol. 49, no. 9, pp. 1520-1533, Sep. 2004.

[16] P. Kokotović, H.K. Khalil, and J. O'Reilly, Singular Perturbation Methods in Control: Analysis and Design. Philadelphia: Society for Industrial and Applied Mathematics, Jan. 1999.

[17] Md. Rasheduzzaman, "Small signal modeling and analysis of microgrid systems," Ph.D. dissertation, Electrical and Computer Engineering, Missouri University of Science and Technology, Rolla, MO, 2015.

[18] Md. Rasheduzzaman, J. A. Mueller, and J. W. Kimball, "Reduced-order small-signal model of microgrid systems," IEEE Trans. Sustain. Energy, vol. 6, no. 4, pp. 1292-1305, Oct. 2015 .

[19] B. Moore, "Principal component analysis in linear systems: Controllability, observability, and model reduction," IEEE Trans. Autom. Control, vol. AC-26, no. 1, pp. 17-32, Feb. 1981.

[20] P. W. Sauer and M. A. Pai, Power System Dynamics and Stability. Upper Saddle River, N.J: Prentice Hall, 1998, Chap. 8

[21] I. Pérez-Arriaga, G. Verghese, and F. Schweppe, "Selective Modal Analysis with Applications to Electric Power Systems, PART I: Heuristic Introduction,' IEEE Trans. on Power Apparatus and Syst., vol. PAS-101, no. 9, pp. 3117-3125, Sep. 1982.

[22] A. Bidram, V. Nasirian, A. Davoudi, and F. L. Lewis, Cooperative Synchronization in Distributed Microgrid Control, ser. Advances in Industrial Control. Cham, Switzerland: Springer International Publishing, 2017, Chap. 3. 